\title{
CO INTERFEROMETRIC MAPS OF CIT 6 AND CRL 618
}

\author{
M. MEIXNER and W.J. WELCH \\ Dept. of Astronomy, U.C. Berkeley, Berkeley,CA 94720 USA
}

We present CO (1-0) interferometer maps of carbon star, CIT 6, and carbon rich proto-planetary nebula, CRL618. Both objects were mapped with the BerekeleyIllinois-Maryland millimeter array (BIMA). The resulting resolution is $\sim 4^{\prime \prime}$; and structure larger than $\sim 1^{\prime}$ is completely filtered out. Two velocity resolutions were employed; $0.4 \mathrm{~km} \mathrm{~s}^{-1}$ and $0.8 \mathrm{~km} \mathrm{~s}^{-1}$ permitting a velocity coverage of 52 and 104 $\mathrm{km} \mathrm{s}^{-1}$. We find evidence for non-spherical expanding envelopes in both objects. The interferometer detected all the single dish CO flux in CRL618 (840 Jy km $\mathrm{s}^{-1}$ ), and $70 \%$ of the CO flux in CIT 6 (or $1030 \mathrm{Jy} \mathrm{km} \mathrm{s}^{-1}$ ). For CIT 6, we obtained single dish maps at the NRAO $12 \mathrm{~m}$ to measure the zero spacing flux distribution. We measure an envelope diameter of $90^{\prime \prime}\left(2.6 \times 10^{17}\right.$ at $\left.190 \mathrm{pc}\right)$ for CIT 6 and $15^{\prime \prime}\left(4.5 \times 10^{17}\right.$ at $\left.2 \mathrm{kpc}\right)$ for CRL 618 and expansion velocities of $17 \mathrm{~km}$ $\mathrm{s}^{-1}$ for both. The small scale structure seen by the interferometer in CIT 6 has a core and an elongation to the SW. We interpret this elongation as the beginning of an equatorial density enhancement and predict that that CIT 6 will evolve into a bipolar planetary nebula. In CRL 618 , the elongated structure seen in $\mathrm{CO}$ is perpendicular to the optical reflection nebula, which has an east-west orientation. Hence the Asymptotic Giant Branch (AGB) wind appears as an expanding waist band around this bipolar nebula giving credence to the idea that equatorial density enhancements in AGB winds produce bipolar planetary nebulae (Balick 1987).

We measure a continuum flux of $1.6 \mathrm{Jy}$ in CRL 618 , confirming previous observations (Martin-Pintado et al. 1988). This continuum arises from the free-free emission in the central HII region which is unresolved in our observations. No continuum is detected in CIT6.

CRL 618 has been observed to have a high velocity molecular outflow (Cernicharo et al. 1989). We have marginally detected this outflow which is unresolved in our observations; and are working on improving the continuum subtraction in order to solidify this detection.

\section{References}

Balick, B.: 1987, $A J$ 94, 671

Cernicharo, J., Guelin, M., Martin-Pintado, J., Penalver, J., and Mauersberger, R.: 1988, AA 222, L1

Martin-Pintado, J., Bujarrabal, V., Bachiller, R., Gomez-Gonzalez, J. and Planesas, P.: 1988, A A 197, L15 